SCIENTIFIC REPORT

\title{
Histopathological analysis in experimental macular surgery with trypan blue
}

\author{
S Grisanti, P Szurman, O Tatar, F Gelisken, S Aisenbrey, J Oficjalska-Mlynczak, R Kaczmarek, \\ K U Bartz-Schmidt
}

Br J Ophthalmol 2004;88:1206-1208. doi: 10.1136/bjo.2004.042101

\begin{abstract}
Aim: To analyse the effect of trypan blue on the retina in an experimental setting of macular surgery.

Methods: Porcine eyes were used within 3 hours after death. The eyes were hemisected and the vitreous removed. Trypan blue $(0.15 \%)$ was applied over the trephined posterior pole, whereas the rest of the eye cup was filled with a balanced salt solution (BSS). The dye and the BSS were removed after 1 minute and the complete eye cup irrigated and filled with fresh BSS. Both the treated and untreated retinas were illuminated with a standard surgical light pipe and source at maximum power for 10 minutes. Both the trypan blue exposed retina and the non-treated surrounding retina were processed for histology.

Results: Exposure of the retina to trypan blue for 1 minute, followed by illumination caused no histologically detectable damage compared to the controls. No microarchitectural disorganisation, cellular disruption, or affection of the vitreoretinal interface was detected.

Conclusions: These findings indicate that a 1 minute exposure of trypan blue followed by illumination does not cause an acute morphologically detectable toxic effect on the porcine retina.
\end{abstract}

$\mathrm{T}$ he vital dye trypan blue has been recently introduced to posterior segment surgery to assist the peeling of the internal limiting membrane (ILM) ${ }^{1}$ and of epiretinal membranes (ERMs). ${ }^{2}$

Trypan blue has a long standing history in anterior segment surgery, where it has been used for vital staining of corneal endothelium ${ }^{3}$ and for the visualisation of the anterior lens capsule in mature white cataract. ${ }^{4}$ Though no negative clinical effects have been reported yet, trypan blue has the potential to induce toxic side effects. ${ }^{56}$ Introduction of this vital dye into posterior segment surgery has been, therefore, anticipated by in vitro and in vivo studies. ${ }^{78}$

Stalmans et al demonstrated that cultured human retinal pigment epithelium cells exposed for 5 minutes to various concentrations $(0.06 \%, 0.15 \%, 0.30 \%)$ of trypan blue did not show increased cell death. Based on these results the authors concluded that a short exposure to trypan blue does not have a toxic effect on cultured pigment epithelium cells. ${ }^{7}$

In order to evaluate the toxic potential of trypan blue in vivo, Veckeneer and co-authors used a rabbit model. New Zealand white rabbits received intravitreal injections of $0.1 \mathrm{ml}$ basic salt solution, $0.1 \mathrm{ml}$ of a $0.06 \%$ trypan blue solution, or $0.1 \mathrm{ml}$ of a $0.2 \%$ trypan blue solution. Electroretinography showed no significant changes in any of the animals. However, the rabbits were sacrificed 4 weeks after the injection and the histological analysis disclosed severe damage of the inferior retina in animals treated with
$0.2 \%$ trypan blue. The authors concluded that trypan blue applied at a concentration below $0.2 \%$ in the vitreous cavity is harmless to the rabbit eye but a higher concentration of trypan blue could be unsafe. ${ }^{8}$

Based on these studies trypan blue at a concentration of $0.15 \%$ became commercially available (MembraneBlue, DORC International, Zuidland, Netherlands) for posterior segment surgery. In order to further evaluate the potential side effects of trypan blue, we mimicked the clinical situation and used trypan blue as recommended by the manufacturer in an experimental ex vivo model of macular surgery.

\section{MATERIALS AND METHODS}

The adult porcine eyes $(n=3)$ were received from the local abattoir, transported to the laboratory on ice, and used within 3 hours after death. After removal of the anterior segment, the vitreous including the vitreous cortex was completely removed and discarded. Conesquently, a trephine of $9 \mathrm{~mm}$ diameter was positioned over the posterior pole and left in place to create a chamber that allowed a standardised application of trypan blue without treating the areas outside of the chamber. The eyecup itself created a second chamber surrounding the trephine. This second chamber was filled with balanced salt solution (BSS; Pharmacia, Groningen, Netherlands) as control (fig lA).

Commercially available trypan blue $0.15 \%$ (Membrane Blue, DORC International, Zuidland, Netherlands) in phosphate buffered sodium chloride $(\mathrm{NaCl})$ was used. The volume used within the trephine chamber was $0.5 \mathrm{ml}$. Therefore, the absolute dose of trypan blue applied to the retina was $0.075 \mathrm{mg}$. The trypan blue solution was poured into the trephine and, as recommended by the manufacturer, left in contact with the retina for 1 minute. The removal of the dye was followed by the removal of the trephine and irrigation of the eye cup with fresh BSS. Similar to clinical practice the probed area showed a faint staining. In consequence, the eye was filled with BSS and the posterior pole was illuminated (10 minutes) with a new standard light pipe (Spectra-Band Fiberoptic Endo-Illuminator, Altomed Ltd, UK) and the fibreoptic light source (50 W; Pentalux, Ruck; Eschweiler, Germany) with no yellow filter set to a maximum illumination power. The fibreoptic was placed $8 \mathrm{~mm}$ above the posterior pole. The illuminated area included both the trypan blue stained area and part of the surrounding control area (fig 1B). Thereafter, retinal specimens from the illuminated untreated area and from the illuminated trypan blue treated area were obtained. The specimens were placed in phosphate buffered $4 \%$ glutaraldehyde solution for fixation. The specimens were then postfixed with Dalton fixative (osmium $2 \%$ ), dehydrated, and embedded in Epon. Semithin sections were stained with toluidine blue for light microscopy. The

Abbreviations: BSS, balanced salt solution; ERM, epiretinal membrane; ILM, internal limiting membrane 

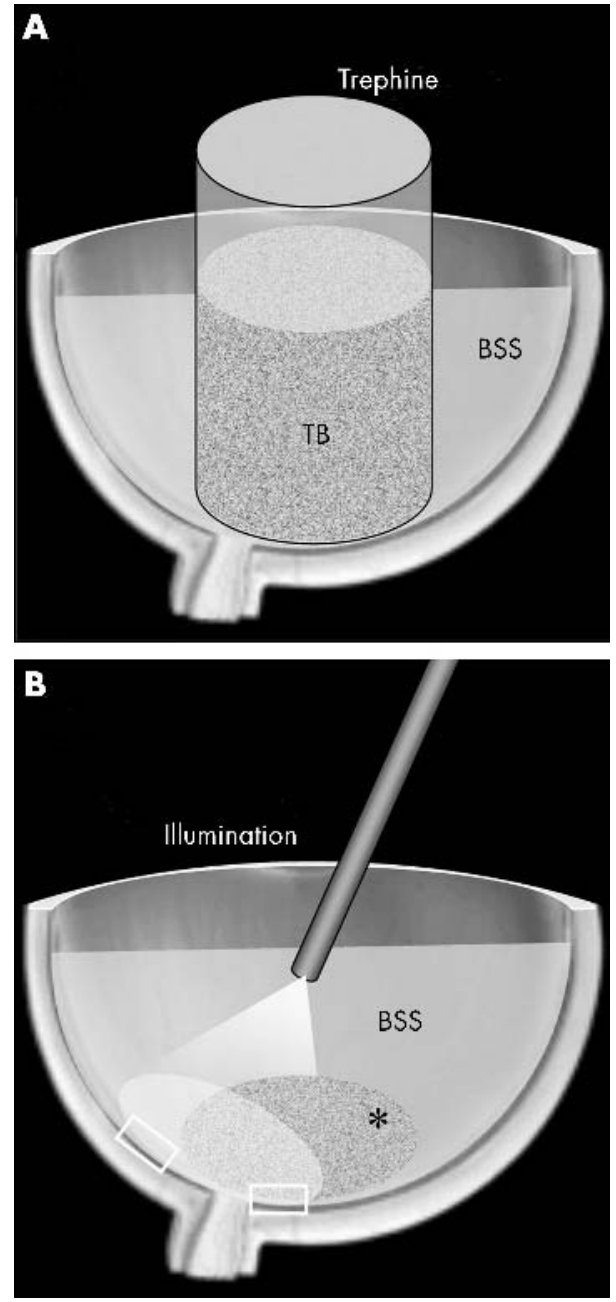

Figure 1 Experimental ex vivo model of macular surgery. (A) A trephine is positioned over the posterior pole and filled with the trypan blue (TB) solution. The rest of the eye cup is filled with a balanced salt solution (BSS). (B) After the removal of the trypan blue solution and the trephine and rinsing of the eyecup with fresh BSS the fibreoptic illuminates both the stained trypan blue treated ${ }^{*}$ and the untreated area. Specimens from both areas ( $\square$ ) are then preserved for histology.

experiment was repeated three times. Osmolarity and $\mathrm{pH}$ of the trypan blue solution were measured on a cryo-osmometer (Osmomat 030, Gonotec; Germany) and a microprocessor pHmeter ( $\mathrm{pH} 211$, Hanna Instruments; Portugal), respectively.

\section{RESULTS}

Representative semithin sections of the treated and untreated specimens are shown in figure 2 . The specimens were evaluated by two observers independently and in a blind fashion. There was no disagreement in their interpretation. All the specimens had a well preserved retinal cytoarchitecture. No disruption of the nerve fibres or of the ganglion cells could be detected in any of the sections. No disorganisation of the cytoarchitecture could be found in any of the specimens. Both nuclear layers and the photoreceptors outer segments were well preserved.

The ILM in porcine eyes is much thinner than in human eyes. However, no disruption of the vitreoretinal interface was seen and the ILM was still adherent both in the control (fig 2A) and in the experimental groups (fig 2B).

A measurement of the osmolarity and $\mathrm{pH}$ of the solution was performed to exclude damage related to these parameters. The trypan blue solution was $292 \mathrm{mOsm} / \mathrm{kg}$ and

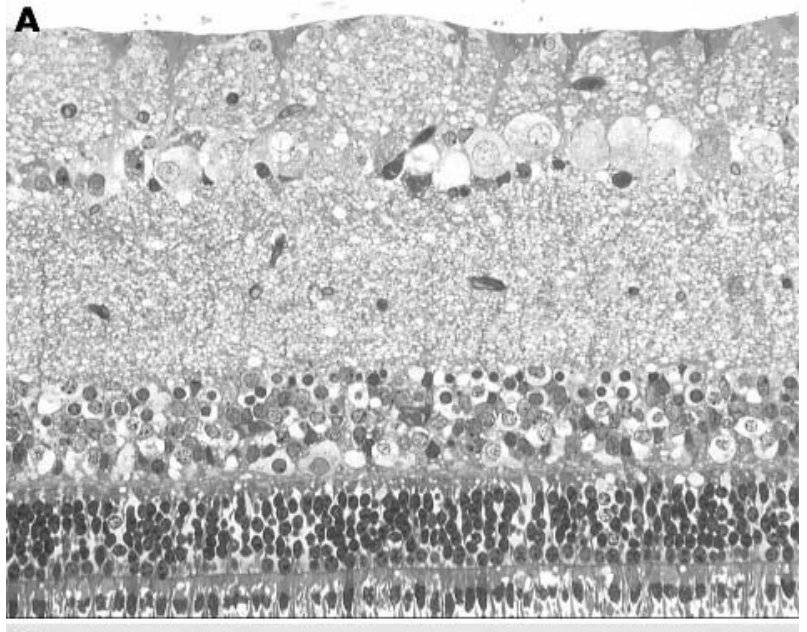

B

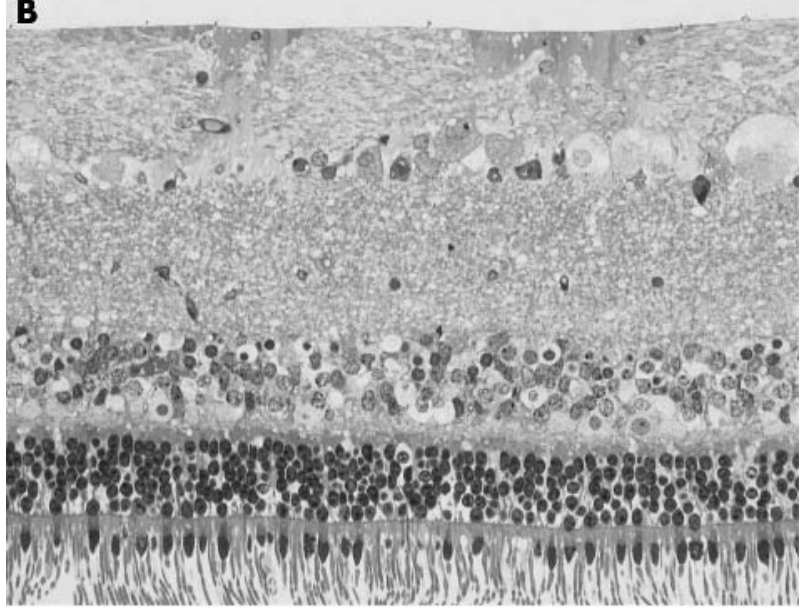

Figure 2 Light microphotographs of toluidine blue stained semithin sections of control $(A)$ and treated $(B)$ retinal specimens taken from the same eye each. Specimens were incubated with BSS (A) or with $0.5 \mathrm{ml}$ $0.15 \%$ trypan blue solution (B) for 60 seconds followed by illumination. All specimens disclosed different degrees of vacuolisation, but showed a well preserved cytoarchitecture and the ILM was attached.

found to be in the iso-osmotic range. The osmolarity of the BSS in the control group was $309 \mathrm{mOsm} / \mathrm{kg}$. The $\mathrm{pH}$ of the trypan blue solution was higher $(\mathrm{pH} 8.1)$ compared to BSS ( $\mathrm{pH}$ 7.2). This difference, however, did not seem to induce morphologically detectable damage in the retina.

\section{DISCUSSION}

Recently, clinical trials ${ }^{129}$ have been published introducing trypan blue as a useful tool to visualise the ILM and ERMs and to facilitate the peeling of these hardly visible tissues. In order to exclude a toxic effect of trypan blue both in vitro ${ }^{7}$ and in vivo ${ }^{8}$ studies have been performed so far.

Veckeneer et al demonstrated in a rabbit model that at a concentration of $0.06 \%$ trypan blue may exert no toxic effects to the retina 1 month after intravitreal injection. ${ }^{8}$ Though, the concentration of the used trypan blue solution $(0.06 \%)$ is lower than the concentration of the commercially available trypan blue $(0.15 \%)$, they could show that a prolonged exposure of $0.06 \%$ trypan blue did not show any electroretinograph or histological damage. Some limitations of this study, however, are that (a) the dye is injected into the vitreous/gas filled eye and (b) the injected volume $(0.1 \mathrm{ml})$ and therefore also the dose of trypan blue is much lower than in clinical practice $(0.5 \mathrm{ml}){ }^{1}$ 
In the study of Veckeneer et al rabbits received $0.4 \mathrm{ml} \mathrm{100 \%}$ $\mathrm{C}_{3} \mathrm{~F}_{8}$ into the vitreous chamber. Three days later (at the time of maximum gas expansion) $0.1 \mathrm{ml} 0.06 \%$ trypan blue was injected into the eye. Since no information was given about the percentage of gas filling of the eye, we assume that in the best of cases a $50 \%$ filling was achieved. This would mean that $0.06 \%$ trypan blue injected into a remaining volume of $1 \mathrm{ml}$ has been further diluted to a significantly lower concentration. In clinical practice, however, a $0.15 \%$ trypan blue solution (Membrane blue) is injected in the air filled globe and may have a stronger impact on the exposed retina. Because of this remaining doubt, we decided to mimic the clinical application of trypan blue during macular surgery as previously described ${ }^{10}{ }^{11}$ in an experimental ex vivo setting. In this model porcine eyes were chosen, because (a) the pig retina closely resembles that of humans, and (b) human donor eyes are not available within 3 hours after death and the autolytic process will influence the results of the study.

In our study, trypan blue caused neither a disorganisation of the retinal cytoarchitecture, nor a cellular disruption. Based on this result we have to assume that the trypan blue solution and the technique of application as is used in clinical practice seem to be safe. However, for the proper interpretation of our results the limitations of this ex vivo study need to be addressed. Firstly, this experimental setting allows only a morphological evaluation of an acute toxic effect of trypan blue. Though the morphology of the examined retinal specimens appeared unaffected by a procedure that is also performed clinically, a functional or delayed effect of trypan blue cannot be excluded. Secondly, though the eyes were processed within a short time after death, the reaction of perfused tissue may well be different. Thirdly, though porcine and human eyes are similar species related differences cannot be excluded.

To summarise, the clinical application of trypan blue seems to be within safe limits. However, an appropriate in vivo model mimicking the surgical procedure and the close observation of the patients submitted to this technique should further support the successful management of trypan blue assisted posterior segment surgery.
Authors' affiliations

S Grisanti, P Szurman, O Tatar, F Gelisken, R Kaczmarek, K U BartzSchmidt, Department of Ophthalmology I, Eberhard-Karls University Tuebingen, Germany

S Aisenbrey, Department of Neuroscience, Tufts University, Boston, MA, USA

J Oficjalska-Mlynczak, Department of Ophthalmology, University of Wroclaw, Poland

Correspondence to: Assistant professor S Grisanti, Department of Ophthalmology I, Eberhard-Karls University of Tuebingen,

Schleichstrasse 12-15, 72076 Tuebingen, Germany;

salvatore.grisanti@med.uni-tuebingen.de

Accepted for publication 29 February 2004

\section{REFERENCES}

1 Li K, Wong D, Hiscot P, et al. Trypan blue staining of internal limiting membrane and epiretinal membrane during vitrectomy: visual results and histopathological findings. Br J Ophthalmol 2003;87:216-19.

2 Feron EJ, Veckeneer M, Parys-Van Ginderdeuren R, et al. Trypan blue staining of epiretinal Membranes in proliferative vitreoretinopathy. Arch Ophthalmol 2002;120:141-4.

3 Norn MS. Peroperative trypan blue vital staining of corneal endothelium. Eight years' follow up. Acta Opthalmol 1980;58:550-5.

4 Melles GR, de Waard PW, Pameyer JH, et al. Trypan blue capsule stainig to visualize the capsulorhexis in cataract surgery. J Cataract Refract Surg 1999;25:7-9.

5 Ema M, Kanoh S. Studies on the pharmacological bases of fetal toxicityof drugs (II). Effect of trypan blue on the pregnant rats and their offspring. Nippon Yakurigaku Zasshi 1982;79:369-81.

6 Chung KT. The significance of azo-reduction in the mutagenesis and carcinogenesis of azo dyes. Mutat Res 1983;114:269-81.

7 Stalmans P, van Aken EH, Melles, et al. Trypan blue not toxic for retinal pigment epithelium in vitro. Am J Ophthalmol 2003;135:234-36.

8 Veckeneer M, van Overdam K, Monzer J, et al. Ocular toxicity study of trypan blue injected into the vitreous cavity of rabbit eyes. Graefes Arch Clin Exp Ophthalmol 2001;239:698-704.

9 Stalmans P, Feron EJ, Parys-Van Ginderdeuren R, et al. Double vital staining using trypan blue and infracyanine green in macular pucker surgery. Br J Ophthalmol 2003;87:713-16.

10 Gandorfer A, Haritoglou C, Gandorfer A, et al. Retinal damage from indocyanine green in experimental macular surgery. Invest Ophthalmol Vis Sci 2003;44:316-23.

11 Grisanti S, Szurman P, Gelisken F, et al. Histological findings in experimental macular surgery with indocyanine green. Invest Ophthalmol Vis Sci 2004;45:282-86 